\title{
CREATING EFFECTIVE ONLINE DISCUSSIONS: OPTIMAL INSTRUCTOR AND STUDENT ROLES
}

\author{
Marcia Dixson, Ph.D. \\ Michelle Kuhlhorst, M.A. \\ Amber Reiff, M.A \\ Indiana University-Purdue University at Fort Wayne
}

\begin{abstract}
Teaching online courses is unexplored territory for many instructors. Understanding how to use the powerful learning techniques involved in cooperative group learning in an online context is imperative. This paper is a first attempt to explore the dynamics of learning groups in the context of online discussion forums. Given the spread of online learning, the importance of this kind of research cannot be overstated. The authors analyzed the content of twenty asynchronous discussion forums within an online course in family communication. Their findings indicate that such groups send social messages at a fairly high rate; that participation seems to be fairly evenly distributed among members, although having a "leader" is helpful to the group's process; and that instructor messages and competitive student messages have no apparent effect on the final product. However, they also found that two types of messagesorienting/giving information and showing solidarity-were found more frequently in groups that produced higher quality work. This research opens many questions and offers some guidelines about the most effective ways to structure online learning situations.
\end{abstract}

\section{KEYWORDS}

Online Discussions, Learning Groups, Cooperative Learning, Asynchronous

\section{INTRODUCTION}

Computer-mediated communication (CMC) has become common in today's classrooms [1, 2, 3, 4, 5, 6]. According to a recent report by the Sloan Foundation [7, p. 1], over 1.6 million students in the United States took an online course in Fall 2002. Over one-third of these students $(578,000)$ took all of their courses online. Advances in technology have created an opportunity that institutions, faculty, and students are eager to use to their advantage. Students readily accept online learning because computers have become part of their lives, and faculty and institutions see it as a way to cope with heavier workloads and shrinking resources.

Reed, Michael, Spuck, and Dennis [3, p. 555] found that sometimes "students rebel when computers are not at their disposal as learning tools," a finding that testifies to the integration of computers in students' lives. Today's students are accustomed to computer use and expect this powerful medium to be effectively utilized to their advantage-both in terms of learning and convenience-within college classes.

From a faculty perspective, Ross and Schulz [4] attribute the increase in instructional technology to larger class sizes and heavier workloads: teachers are looking toward computers as a means to supplement classroom instruction because of the computer's capacity to individualize instruction to meet the specific needs of the learner. 
In short, online learning gives both teachers and students access to shared resources and information and a means to communicate with others on both a national and an international level [6]. Given that online education is probably here to stay, it remains for researchers to explore how the medium can be most effectively applied.

This study contributes to the exploration process by looking at the use of small group discussion as a learning tool within the online environment. After a brief discussion of the advantages and disadvantages of online learning, we describe what the research indicates regarding what instructors can do both online and in traditional classrooms to create effective learning discussions. Then we investigate the possible effects of student behavior on learning, first through a review of the literature and then the study.

\section{A. Advantages and Disadvantages of Online Learning}

Possibly one of the most important aspects of online learning is how the students themselves perceive the online learning experience. Because these types of courses are fairly new, valuable insight from the students can benefit future design of online courses. Research indicates that students are in favor of online courses; in two separate studies, students listed such benefits as flexible scheduling, ability to work any time and at any place, and ability to choose the best conditions for learning. They also mentioned saving gas and time commuting, having more access to the instructor and to their fellow classmates, and being able to attend to family responsibilities and still take courses as other benefits of the online experience [8, 9]. In another study, students liked the fact that they could post their assignments electronically and review their peers' work, because in doing so they learned more and were motivated to help each other out [10].

However, students also mentioned several perceived disadvantages in online courses compared to the traditional classroom environment. The students missed face-to-face communication and personal contact. Students with low technology skills felt pressured and anxious, which could have a negative impact on learning [8]. Many students noted that self-discipline was necessary to success in an online course. Despite these barriers, students in the Logan, Augustyniak, and Rees study [11] said that they would still choose an online course over a traditional course if given the choice.

To overcome some of the drawbacks (such as the lack of personal contact and face-to-face communication) and to take advantage of some of the benefits (such as the ease of participation because of the relative anonymity of the web [11]) many online instructors are attempting to move small group discussion-also called cooperative or team learning-from the traditional classroom to the online environment. The benefits of team-based or group learning in the traditional classroom have been documented by such theories as Bandura's observational learning [12] and Vygotsky's notion that higher order thinking originates in social interaction that is subsequently internalized [13].

Likewise, recent research supports students' desire for interaction among students and between students and instructors in web-based courses. For instance, Arbaugh and Hornik [14], in a study of 385 students in online sections of MBA programs, found that student-faculty contact was moderately associated with perceived learning and strongly associated with course satisfaction, and that collaboration was positively associated with learning. Shea, Frederickson, Pickett, and Pelz [15] found that students in courses with instructor-student dialogue were enthusiastic about their experiences, whereas students without such dialogue were frustrated and angry. These researchers also found that $86 \%$ of the 935 SUNY Learning Network students reported putting more thought into online discussion postings than they would in a faceto-face classroom discussion. Students also reported being twice as likely to participate in an online 
discussion as a classroom discussion [9]. Thus, not only do online students desire interaction but they may put more thought and effort into those interactions.

Current course management programs (e.g., Blackboard, WebCT) offer a way to use group interaction in the virtual classroom through discussion forums, where students can post comments and replies to an initial posting on a central topic by the instructor or a student. Many courses use this asynchronous form of small group discussion because, unlike a chat room, it does not require all members of the group to be online at the same time. Thus, asynchronous discussions meet one of the primary criteria for students choosing distance learning: the convenience of not always needing to be in class at a given time. To further understand online learning's capacity to support small-group discussion, we reviewed the literature on what instructors can do in both a traditional and an online classroom, and what students do (or should do) in both settings, to render such discussions more effective. What we found is that there is much written about what instructors should do and little about the process occurring between students within these groups.

\section{B. What Instructors Need To Do To Promote Good Group Discussions}

Recently, Larry Michaelsen [16], author of numerous articles on team-based learning and co-author of the book Team-Based Learning: A Transformative Use of Small Groups in College Teaching [17], has identified three primary elements necessary to make team or group learning work: (1) promote ongoing individual and group accountability; (2) use the three $S$ 's - same problem, specific choice answers, and simultaneously reporting; and (3) adopt practices that stimulate idea exchange. Grading or awarding points for both individual and group efforts-individual to curtail social loafing and group to create an interdependent team - can support the first element; the second element concerns stimulating productive exchange between groups after group projects are completed in order to debrief the learning. For the third element, Michaelsen suggests that we use assignments requiring group interaction (presumably these are structured so that individuals cannot complete them alone); remove barriers to participation (he suggests fostering cohesion through permanent groups and choices of assignments and grading practices); use inclass group work so students have time to meet; and create diverse groups to expose students to new ideas. These suggestions are useful and have been found to be successful, at least in the traditional classroom. However, they mostly deal with what the instructor can do, which makes sense given Michaelsen's audience. Once these elements are in place, what can the students do to make the group effective or ineffective?

Researchers have been looking for ways to enhance discussion and in particular to encourage active roles in students [18]. McComb [18] suggests that discussions should be a responsibility, perhaps one that is graded, and that the question starting the discussion needs to be more difficult and require more thinking. He also suggests that groups be no larger than seven and allow only enough time to get the job done.

Another set of well-known learning group researchers has explored what students can do to achieve best results in classroom discussion. Johnson, Johnson, and Smith [19] have theorized that it takes five elements to maximize success for cooperative learning groups: (1) positive interdependence, (2) face-toface interaction, (3) individual accountability, (4) social skills, and (5) group processing. Of these, the first and third sound very much like Michaelsen's ideas and have to be structured by the instructor. The second, face-to-face interaction, is what this study proposes to challenge. Number five, group processing, involves the group engaging in self-reflection in order to fine-tune the group efforts and see when and how they are being effective or ineffective. This element presumes permanent or semi-permanent groups. Assuming that the fifth activity could be assigned by the instructor, it is really the fourth activity, social skills, that deals primarily with what students have to do once the instructor has done all he or she can. 
Let's investigate these social skills a bit more.

Johnson, Johnson, and Smith [19] define social skills as (1) mutual knowledge, which can be arranged by the instructor with a previous assignment, usually a reading, and some kind of readiness assessment tool such as a quiz or quick class activity to assess their individual knowledge of the reading; (2) trust, which has to be built over time; (3) effective communication; and (4) the ability to solve conflicts. But what kinds of effective communication help the group to achieve success? What abilities are needed to solve conflict? Now we reach the crux of what students need to do.

\section{What Students Need To Do}

In a qualitative analysis of high school students' journal and thesis writing about class discussions in an English course, Connolly and Smith [20] identified four themes for the analysis of student discussions. The first one deals with who gets to speak. In online classes, anonymity is protected, and the fear of rejection is decreased in electronic discussions to the point at which students may feel less intimidated to speak up [11]. Often students only know each other's names; any other information (including that usually gained from nonverbal clues) has to be explicitly disclosed. Many researchers note that students perceive online discussions as more equitable and more democratic than traditional classroom discussions [10]. We were interested in seeing if a dominant class member(s) would emerge in this environment. If one (or more) did, does this affect the learning of the group, at least as can be seen by the quality of the product the group creates? This line of inquiry led to our first research question and follow-up question:

$\mathrm{RQ}_{1}$ : Are online conversations evenly distributed among members? If not:

$\mathrm{RQ}_{1 \mathrm{a}}$ : Is there a relationship between having a dominant member in the group and the quality of the final product of the group?

The second theme Connolly and Smith identified is the way students speak: one-way or two-way. For example, do the students in discussion have a common goal or a goal of having the last word? One quarter of students in the Connolly and Smith study felt that competition is a positive characteristic to have in discussions, whereas the instructor in the study said it was not [20]. Our second research question explores this use of competitive messages, again by relating it to the learning evident in the final product:

$\mathrm{RQ}_{2}$ : Do discussions with more competitive messages create a better product?

Connolly and Smith's [20] third theme deals with what the students will talk about. According to their research, the students' relationship with the instructor influences subject matter. If the students feel close to the instructor, chances are that the conversations will be more provisional than direct. The last theme deals with the arrangement in which the conversation is taking place. High school students in the Connolly and Smith study reported preferring smaller groups with higher intimacy because of their fear of ridicule. We believe we still see this in the university classroom. We did not test these themes but held them constant by choosing to use small groups of four to five people in a course in which the instructor's student evaluations consistently rate her as "highly approachable."

However, we did want to look at the kinds of messages students send to see if any are consistently related to higher quality products (and, thus, the potential for more learning). For instance, is it more important to have people clarifying ideas, asking questions, or offering information? Which of these leads to a better final product in terms of the group's "final answers" to the questions? Therefore, our third research question explores functions of messages and their relationship to the groups' final products.

$\mathrm{RQ}_{3}$ : What kinds of messages are likely to lead to better products of the online group? 
The benefits of utilizing an asynchronous learning environment include collaboration and cooperation. Students should be encouraged to offer information and insights from their own experiences, allowing them to gain information and insights from other members of the group as well as the instructor. Social interaction with their instructors and collaborative interaction with peers are important to enhance learning and increase participation in online discussions. A study conducted by Gunawardena and Zittle [21] revealed that social presence contributed more than $60 \%$ of learner satisfaction with computer conferencing courses.

Because social messages are an important part of group maintenance and cohesion, we also wanted to see if students do, in fact, send social messages in this online environment. According to the literature, the students should be comfortable enough to have conversations for non-school-related purposes because physical and demographic characteristics (race, religion, sexual orientation, and so forth) are not as easily discovered on the web as in face-to-face communication [11]. This relative anonymity should make students more likely to engage in social conversation.

$\mathrm{RQ}_{4}$ : Do students send social messages in online groups?

Interaction has been recognized as one of the most important components of learning experiences both in conventional education and distance education [22]. This interaction is important for not only peer-to-peer communication but also student-to-instructor communication. The instructor has to undertake many tasks before, during, and after the sessions to ensure that good discussions will ensue. These include planning the collaborative process, intervening to ensure positive and beneficial behavior, encouraging learning and changes in perceptions by utilizing the appropriate techniques, and acquiring feedback from the learners about the process and content of the course [23]. Instructors need to be sensitive about such issues as when to intervene, withdraw, or enter the dialogue; they have the responsibility to keep the discussion going by evoking responses, questioning, interpreting, synthesizing, advocating, serving as devil's advocate, mediating, providing information, reformulating questions, amplifying, and maintaining discussions through questions and explications [18, p. 167]. The instructor must also invite group members to present their own knowledge, experiences, and ideas through group cohesion and attention to the task. Given this formidable charge, we were interested in knowing what instructor messages might have the most impact on learning as measured by the groups' final products.

$\mathrm{RQ}_{5}$ : What kinds of instructor messages lead to better group discussion products?

Interaction among students through course discussions seems to be one of the most influential features of online courses [24, 25]. Computer-mediated communication encourages experimentation, sharing of ideas, increased and more distributed participation, and collaborative thinking. To be successful, online discussion requires a social environment that encourages peer interaction facilitated by the structured support of the instructor. Swan et al.'s study about consistency and communication in the virtual classroom indicates that online courses that are both well structured and easy to use and that take advantage of increased access to instructors [24] and more equitable and democratic discussion are the most successful [23]. We set out to explore how that environment might be accomplished using the small group discussion forum.

\section{METHODS}

\section{A. Participants}

Participants were students in a family communication course taught entirely via the Internet. Five group discussions were held in discussion forums within the course management program (WebCT). Six groups 
consisting of four to six people each discussed the five topics. Most of the discussions centered on case studies and scenarios that students had to analyze according to specific models or concepts from the text. For instance, the story of Ruth Steinem's battle with mental illness would be analyzed to understand the possible influences of societal/cultural expectations of the time. The class consisted of four males and 23 females: eight sophomores, eight juniors, and eleven seniors. Student majors were general studies (nine students), business and organizational leadership (seven students), health sciences (three students), sociology (three students), two undecided majors, and one each of criminal justice, communication, and human services. In short, students across the curriculum participated in the class. Because it is an online course, no other demographic information was available.

\section{B. Procedure}

The group assignments for this study were awarded both an individual (based on participation) and a group (based on the final product) grade, an approach that created both individual and group accountability. Likewise, one or two reading assignments and a quiz preceded each assignment to ensure that everyone in the group was up to speed on the necessary information. All groups undertook the same assignment, and all were required to post their final answers on the same day. The final answers forum is the only one that was accessible to the entire class; each of the other group discussion forums was accessible only to the members of one group. As Michaelsen [16] recommends, the class used permanent groups (called families) that worked together throughout the semester. The goal of the first, ungraded discussion was to create a name for the family, thus allowing students to become familiar with the technology before starting a graded discussion. Groups were randomly generated by WebCT and thus were probably as diverse as possible given the generally homogeneous nature of students, except as pertains to age (ages range from 14 to 70 with an average age of 25.6 [26]). Because of the wide age range of the students, a good measure of relevant diversity was present in this class. The course generally has students from diverse family backgrounds and structures, and this diversity is helpful to the discussion. The two areas that need further work, from the instructor's perspective, were a debriefing activity with the entire class once the discussion was over to compare and contrast group answers, and a time for the groups to review and analyze their own processes, perhaps at midterm. So, while some improvements could still be made to the structure of the discussions, most of the expert recommendations on the creation of a rich learning environment were followed.

Each of the thirty discussion forums (six groups with five discussions each) was downloaded from WebCT. All names were then replaced with codes that allowed researchers to know when a given individual was speaking but removed all identifying information. Two groups were excluded from the coding because they consistently decided to hold their group discussions in a chat room. Although the chat rooms allow for downloading logs, this medium was beyond the scope of this study.

The remaining twenty group discussions (19 students) were coded using an adaptation of Bales' interaction process analysis (IPA) system (Bales, 1948, as cited in Reinard [27]). Discussions consisted of a total of 297 postings. The unit of analysis was the thought unit; one unit was coded each time a participant seemed to have a particular point to make or complete thought to convey. For instance, an analysis of a conflict scenario might include a statement such as "Joe is stonewalling Danielle," and this statement would be considered one thought unit. A statement such as "Men and women communicate differently, and we must recognize these differences in order overcome some of these challenges" would be considered two thought units since it represents two ideas. There were 1,057 thought units coded.

After determining the units to be coded, the coders decided in which of Bales' twelve categories each unit belonged. Bales designed the categories for content analysis of verbal messages in problem-solving group 
discussions, and the system adapts well to the postings of discussion forums. The IPA consists of six categories that affect the social/relational aspects of the group: three that are broadly considered positive (shows solidarity, shows tension release, and agrees) and their opposites (shows antagonism, shows tension, and disagrees). The IPA's other six categories are considered task categories (gives suggestion, gives opinion, and gives information) and their opposites (asks for suggestion, asks for opinion, and asks for information). The adaptations we made were to clarify the categories for use in the discussion forum. For instance, Category 4 (gives suggestion) and Category 9 (asks for suggestion) were used only with procedural issues of how to proceed with the assignment, clarifying objectives for the assignment, and so forth. Categories 5, 6, 7, and 8-giving or asking for opinion, giving or asking for information-were assigned for messages about the task itself (not how to proceed with the task).

Because we were interested in how disagreement, asserting one's own opinions, and competition might affect learning and the final product, we created a dummy category of competitive messages. Competitive messages were those coded into Categories 5 (gives opinion), 10 (disagrees), or 12 (shows antagonism). The entire coding manual can be found in Appendix A.

Two graduate students were trained to code using dummy data until they achieved .95 intercoder reliability. These coders proceeded to code the discussion forums. Periodically, the coders recalibrated by coding the same set of data and checking intercoder reliability. Any drop below .90 resulted in further clarification of the coding manual and/or retraining of the coders with further recalibration until the .90 level was again reached.

The quality of the final product (answers reached by groups) was evaluated by the course instructor/ primary investigator without knowledge of any of the coding results. Each final posting was evaluated on a four-item scale consisting of (1) how relevant the answer was to the assignment, (2) how well the group supported their answer, (3) how insightful of family communication dynamics the answer was, and (4) how well they applied the relevant family concepts. Each item was evaluated on a scale of 1 (strongly disagree) to 7 (strongly agree). A Cronbach's alpha of .73 was achieved for this scale.

\section{RESULTS}

$\mathrm{RQ}_{1}$ : Are online conversations evenly distributed among members?

Chi-square tests run on the number of messages (thought units) by each group member in each group discussion indicated that thirteen groups could be considered to have dominant members (see Table 1 for a complete listing of chi-squares and significance values).

Table 1. Occurrence of Dominant Members in Discussion Groups

\begin{tabular}{|l|l|l|l|l|l|}
\hline \multicolumn{6}{|l|}{ Chi-square to test for dominant members. } \\
\hline \multicolumn{1}{|c|}{ Groups } & \multicolumn{1}{|c|}{ Topic One } & \multicolumn{1}{|c|}{ Topic Two } & \multicolumn{1}{|l|}{ Topic Three } & \multicolumn{1}{|c|}{ Topic Four } & \multicolumn{1}{|c|}{ Topic Five } \\
\hline One & $X^{2}=27.49$ & 25.88 & 26.41 & 25.23 & 19.61 \\
& $\mathrm{p}<.0001$ & .0001 & .0001 & .0001 & .001 \\
\hline Two & $X^{2}=1.31$ & 2.68 & 24.57 & 2.70 & 6.74 \\
& $\mathrm{p}<.52$ & .44 & .0001 & .26 & .03 \\
\hline
\end{tabular}




\begin{tabular}{|l|l|l|l|l|l|}
\hline Three & $X^{2}=26.61$ & 10.42 & 2.27 & 3.26 & 13.83 \\
& $\mathrm{p}<.0001$ & .015 & .13 & .35 & .0003 \\
\hline Four & $X^{2}=22.56$ & 10.67 & 2.12 & 10.59 & 3.09 \\
& $\mathrm{p}<.0001$ & .03 & .71 & .03 & .54 \\
\hline
\end{tabular}

$\mathrm{RQ}_{1 \mathrm{a}}$ : Is there a relationship between having a dominant member in the group and the quality of the final product of the group?

A $t$ test was then run on the final answer scores of the thirteen group discussions with dominant members $(M=19.32 ; s d=3.19)$ as compared to the seven without dominant members $(M=16.28 ; s d=1.10)$. The $t$ test yielded a significant $\underline{\mathrm{t}}_{18}=2.02 ; p=.058$. Having a dominant member produced better end results.

$\mathrm{RQ}_{2}$ : Do discussions with more competitive messages create a better product?

For this question, we tested the dummy category previously created for competitive messages. The number of competitive messages in each group discussion ranged from 3 to 54, with a mean of 18.3. More importantly, the percentage of competitive messages ranged from 23\% to 50\%, with an average of $33 \%$. Therefore, two categories were created: the one with low competition had 31\% or fewer competitive messages, while the high competition category had $32 \%$ or more (there were 10 discussions in each category). A $t$ test run on final scores yielded an insignificant difference $\left(t_{18}=-.93\right)$. Competitive messages did not help groups create a better final answer.

This finding could be attributed to the fact that competitive messages in these discussions (messages of disagreement, antagonism, etc.) tended to be very mild in nature. For instance, a message of disagreement was often preceded by agreement, such as: "I agree with A's [name deleted] definition of (B) with the exception that...”

$\mathrm{RQ}_{3}$ : What kinds of messages are likely to lead to better products from the online group?

To investigate this question, two groups of ten were again formed based on whether the groups had strong or acceptable answers. Since the mean score on postings of final answers was $18.2(s d=3.34)$, the ten group discussions with strong answers had scores at or above the mean of 18; those with acceptable answers had scores below 18. $F$ tests were then run on all twelve message categories to see if any message types were significantly more likely to be found in discussions with strong as opposed to acceptable answers. Only two categories were found to be significantly more prevalent in discussions that produced strong answers. Category 1 (shows solidarity), the third largest category with over $10 \%$ percent of the thoughts posted (146/1057), yielded an $F_{1,18}$ of $4.39(p<.05)$. Comments such as "I feel that this was still very productive for us. Good job Group!!! :)” were important to learning, possibly motivating the group to extra effort.

Category 6, (gives orientation, information, etc.) yielded an $F_{1,18}=3.03(p<.10)$. It makes sense that information presented from text or other resources, such as "Over $65 \%$ of communication is nonverbal," helped anchor the discussions in facts rather than in students' opinions on the family situations and case studies presented.

Although it accounted for almost one-third of the thoughts posted (335/1057), Category 5 (gives opinion, analysis, evaluation) - the largest category—did not significantly affect products. Nor did Category 4 (gives suggestions, direction), the second largest category. 
$\mathrm{RQ}_{4}$ : Do students send social messages in online groups?

Four of Bales' categories are considered social. Categories 1 (shows solidarity; third largest category as stated above), 2 (shows tension release), 11 (shows tension), and 12 (shows antagonism) were combined to create a social messages category. While these are not all positive messages, they convey the relational maintenance functions of the group. The number of social messages per group discussion ranged from 0 to 25, with a mean of 10.5 . More importantly, the percentage of social messages for each discussion ranged from $0 \%$ to $36 \%$, with an average of $19 \%$. These numbers indicate that this research question can be answered in the affirmative.

Social messages sometimes showed support and solidarity for other students as they experienced their own family situations. Comments such as "You are definitely in the middle of the ABC-X Stress model yourself. How is your mom? Your daughter? Did they figure out what is wrong with your daughter?" indicate that students took time to inquire about the personal lives and problems of other students, even though many of these individuals had never met face to face.

$\mathrm{RQ}_{5}$ : What kinds of instructor messages lead to better group discussion products?

Instructor postings were also coded. For this question, the instructor codings were separated out and counted for each of the twenty discussions. Six message categories had a frequency of more than one. These categories were 1 (shows solidarity), 3 (agrees), 4 (gives suggestion), 5 (gives opinion), 6 (gives orientation), and 9 (asks for direction). The same kind of analysis as discussed in $\mathrm{RQ}_{3}$ was run on instructor messages. None of the categories showed a significant difference between groups with strong final answers and groups with acceptable final answers.

\section{CONCLUSIONS}

Although we recognize that further replication is needed and that this was a very limited study, there were some important and interesting findings from this research.

The finding that group discussions with dominant members tended to have higher scores on their final answers indicates that online groups, like face-to-face groups, need leadership as long as everyone is invited to participate. The asynchronous nature of the forums makes it difficult for any member to cut off another member, and each member can "talk" as long as he or she likes. Thus it is unlikely that such members stifled the participation of other group members, a clear advantage for this particular medium. It would be interesting to further analyze these results to see if the same group member tended to be dominant in all discussions or if that role changes with different topics. Because for these assignments each member is required to take a turn at posting the first discussion message and facilitating the discussion, such a change is certainly conceivable. It is interesting to note that only one group had a dominant member or members in all five discussions. This finding seems to indicate that, for the other three groups in this study, dominance may be more a function of the topic or the assigned role than of personality. Pursuing this line of inquiry is certainly an avenue for future research. In the meantime, it may be beneficial for instructors to assign someone to lead or facilitate online group discussions.

Online members may feel more able to participate because of the relative anonymity involved. If so, they are likely to learn more from the exercise. Students know each other's names, but they have no other information about each other besides what they choose to share. It may also be that the ability to respond from the security of their own homes (or the anonymity of the computer lab) emboldens members. Or, just as likely, the more stringent assessment of individual accountability afforded by the online medium may motivate students to contribute. In a discussion forum, the instructor can see exactly what each 
member has contributed and grade accordingly, something not possible in a traditional classroom where all six groups are generally meeting at the same time in class or meet in various places and at many times outside of class.

It would seem that members do socialize in online groups in a fashion similar to that of face-to-face groups. Such social messages help a group become cohesive, creating higher member satisfaction and the possibility of better products. It is interesting that there were more competitive messages than social messages; however, since competitive messages included disagreeing and the offering of differing opinions, this mostly constructive conflict would seem to lend itself to quality group discussions. The fact that there was no effect seen from competitive messages on the product of the group may be the result of small sample size or simply the fact that competitive messages-along with orienting and other types of messages - are all necessary to create a quality final answer.

One of the most interesting findings was the identification of which categories were related to higherquality final products. The finding that giving orientation and information online was found more frequently in groups with stronger final answers is not surprising. The more information the entire group shares, the more likely they are to have the relevant information to create a good decision. However, the finding that showing solidarity led to better decisions was interesting and underscores the importance of the social relationship involved in group dynamics. This finding concurs with other recent research on the importance of solidarity, cohesion, and community to effective online learning [28, 29, 30, 31]. The fact that this social relationship is present and related to better group products in online discussions is heartening to those of us who teach online. The substitution of asynchronous discussion forums for faceto-face interaction is a leap of faith for many instructors. Our evidence indicates that that leap is in good faith.

The final finding must be somewhat disappointing for instructors. The fact that nothing the instructor posted led to any significant difference in the quality of the group's final answer leads us to wonder how important it is for the instructor to directly intervene in online discussions. On the other hand, given that most instructor postings were in the categories of offering information about the assignment, the procedure, or the direction of the discussion, it may be that instructors exercise an indirect effect by ensuring that members are on the right track. This finding thus supports previous research regarding the importance of effective instructional design and organization but does not support recent findings regarding the importance of directed facilitation of student discourse for online learning [28, 29]. Clearly more research on various types of instructor messages should be done. However, if instructors have done their jobs well by creating rich learning environments with clear goals and directions, our role may well be one of ensuring that students are heading in the right direction, and too much "shepherding" may inhibit learning.

More research is called for to determine what kind of instructor interventions, if any, are positive and which may have little to no effect and not be worth the instructors' time. Discovering which instructor interventions are most productive would be worthwhile since online classes seem to take more time to conduct than traditional classes, despite the belief that instructors can use them to offset dwindling time and resources. The better we can use that time by eliminating activities that are not effective, the more time we have for other aspects of the course.

There are many limitations to this research. The small sample size-only one class with one instructoris a problem. On the other hand, twenty discussions seem like a reasonable number for an initial exploration of the topic. Clearly it would be preferable for someone other than the instructor/researcher to 
rate the final answers, although the evaluator should be someone conversant with the course content matter and the objectives of the discussion assignments. While there are numerous ways to improve this study, it does offer a solid beginning for exploring online learning discussion forums.

Apart from the previously discussed advice from the literature on creating discussion forums (such as individual and group accountability, prior individual preparation, all groups working on the same problem and posting answers simultaneously, and an instructor who is considered approachable), there are some other things instructors can do or encourage students to do in order to create a rich learning environment. Recalling, of course, that these conclusions are drawn from a very limited sample, we offer a few suggestions. Teachers should encourage a relatively informal environment that will promote social messages within the discussion forum. A leader should be appointed to facilitate group discussions. Individual accountability that requires all members to participate with their own ideas and information should be encouraged and is probably worth the time it takes for instructors to assign individual grades. Then, beyond checking in to see that groups understand the assignment and are on track, instructors should get out of the way and let students learn!

Research into online discussion forums offers not only the possibility of understanding what is happening in this relatively new teaching and learning medium but also a new arena for researching group dynamics in general. This arena allows for complete access to group messages without the intrusion of the researcher into the group context. Given the rapid growth of online teaching, further investigations into how best to use this medium for the maximum benefit of students would be both important and timely.

\section{REFERENCES}

1. Ester, D. P. CAI, lecture, and student learning style: The differential effects of instructional method. Journal of Research on Computing Education 27(2): 1-9, 1995.

2. Jaffe M. J., Y. Lee, L. Huang and H. Oshagan. Gender identification, interdependence, and pseudonyms in CMC: Language patterns in an electronic conference. The Information Society: 221223, 1999.

3. Reed, M., D. Spuck and W. Dennis. Summary of special issue on assessing the impact of computer based learning since 1987. Journal of Research on Computing Education 28(4): 554-558, 1987.

4. Ross, J. and R. Schulz. Can computer-aided instruction accommodate all learners equally? British Journal of Educational Technology 30(1): 5-24, 1999.

5. Rutherford, D. and W. J. Lloyd. Assessing a computer-aided instructional strategy in a world geography course. Journal of Geography in Higher Education 25(3): 341-355, 2001.

6. Selwyn, N. Creating a "connected" community? Teachers' use of an electronic discussion group. Teachers College Record 102(4): 750-778, 2000.

7. Allen, I. E. and J. Seaman. Sizing the Opportunity: The Quality and Extent of Online Education in the United States, 2002 and 2003. Needham, MA: The Sloan Consortium, 2003.

8. Leh, A. Action research on hybrid courses and their online communities. Education Media International 39: 31-39, March 2002.

9. Shea, P. J., K. Swan, E. E. Fredericksen and A. M. Pickett. Student satisfaction and reported learning in the SUNY Learning Network. In: Bourne, J. and Moore, J.C. (Eds.), Elements of Quality Online Education, 145-155. Needham, MA: The Sloan Consortium, 2002.

10. Swan, K. Building learning communities in online courses: The importance of interaction. Education Communication Information 2: 23-50, May 2002.

11. Logan, E., R. Augustyniak and A. Rees. Distance education as a different education: A studentcentered investigation of distance learning experience. Journal of Education for Library \& Information Science 43(1): 32-43, 2002. 
12. Bandura, A. Influence of models' reinforcement contingencies on the acquisition of imitative responses. Journal of Personality and Social Psychology 1(6): 589-595, 1965.

13. Wertsch, J. V. Vygotsky and the Social Formation of Mind. Cambridge, MA: Harvard University Press, 1985.

14. Arbaugh, J. B. and S. C. Hornik. Predictors of perceived learning and satisfaction in web-based MBA courses: A test and extension of Chickering and Gamson's (1987) seven principles of good practice in education. Proceedings of the $33^{\text {rd }}$ Annual Meeting of the Decision Sciences Institute. Atlanta, GA: Decision Sciences Institute, 2002.

15. Shea, P. J., E. E. Fredericksen, A. M. Pickett and W. Pelz. Faculty development, student satisfaction, and reported learning in the SUNY Learning Network. In: Duffy, T. M., and Kirkley, J. R. (Eds.), Learner-Centered Theory and Practice in Distance Education: Cases from Higher Education, 343-377. Mahwah, NJ: Lawrence Erlbaum Associates, 2004.

16. Michaelsen, L. K. Three keys to using learning groups effectively. Essays on Teaching Excellence 9(5): 1998.

17. Michaelsen, L. K., A. Bauman-Knight and L. D. Fink. Team-Based Learning: A Transformative Use of Small Groups in College Teaching. Sterling, VA: Stylus Publishing, 2004.

18. McComb, M. Benefits of computer-mediated communication in college courses. Communication Education 43(2): 159-170, 1994.

19. Johnson, D. W., R. T. Johnson and K. A. Smith. Active Learning: Cooperation in the College Classroom. Edina, MN: Interaction Book Company, 1991.

20. Connolly, B. and M. W. Smith. Teachers and students talk about talk: Class discussion and the way it should be. Journal of Adolescent \& Adult Literacy 46(1): 16-27, 2002.

21. Gunawardena, C. and F. Zittle. Social presence as a predictor of satisfaction within a computer mediated conferencing environment. American Journal of Distance Education 11: 8-26, 1997.

22. Choi, I., C. Lim and J. Leem. Effects of different types of interaction on learning achievement, satisfaction and participation in web-based instruction. Innovations in Education and Teaching International 39: 153-162, May 2002.

23. McFadzean, E., and J. McKenzie. Facilitating virtual learning groups: A practical approach. Journal of Management Development 20(6): 470-495, 2001.

24. Swan, K., P. Shea, E. Fredericksen, A. Pickett, W. Pelz, and G. Maher. Building knowledge, building communities: Consistency, contact and communication in the virtual classroom. Journal of Educational Computing Research 23: 389-413, 2000.

25. The Connecticut Distance Learning Consortium. Teaching tips: What online students want to tell faculty. 2003. http://www.ctdlc.org/Faculty/TeachingTips/tellfaculty.html.

26. Office of Institutional Research, Indiana University-Purdue University Fort Wayne. 2005-2006 Statistical Profile. November 2005. http://www.ipfw.edu/ir/pdfs/Statsprof05-06.pdf.

27. Reinard, J. Introduction to Communication Research. 3rd edition. Boston: McGraw-Hill, 2001.

28. Shea, P., E. Fredericksen, A. Pickett, and W. Pelz. A preliminary investigation of teaching presence in the SUNY Learning Network. In J. Bourne and J. C. Moore (eds.), Elements of Quality Online Educations: Practice and Direction, 279-312. Needham, MA; Sloan-C, 2003.

29. Shea, P., C. Li, K. Swan and A. Pickett. Developing learning community in online asynchronous college courses: The role of teaching presence. Journal of Asynchronous Learning Networks 9(4): 2005.

30. Rovai, A. P. A preliminary look at structural differences in sense of classroom community between higher education traditional and ALN courses. Journal of Asynchronous Learning Networks 6(1): 4156, 2002.

31. Rovai, A. P. Development of an instrument to measure classroom community. The Internet and Higher Education 5: 197-211, 2002. 


\section{ACKNOWLEDGEMENTS}

A previous version of this paper was delivered at the 2004 convention of the National Communication Association in Chicago, Illinois.

\section{ABOUT THE AUTHORS}

Marcia D. Dixson is Associate Professor and Graduate Director of Communication at Indiana University-Purdue University in Fort Wayne, Indiana. She is past director of the campus Center for the Enhancement of Learning and Teaching and began teaching online courses just three years ago. She enjoys the new challenges that online teaching brings. Her research interests include the scholarship of teaching (especially online teaching) and family communication.

Michelle Kuhlhorst recently graduated with her master's degree in professional communication from Indiana University-Purdue University in Fort Wayne, Indiana. She is currently working in public relations for Kruse International in Auburn, Indiana.

Amber Reiff recently graduated with her master's degree in professional communication from Indiana University-Purdue University in Fort Wayne, Indiana. She is currently teaching in California.

\section{APPENDIX A: MODIFICATION OF BALES' INTERACTION ANALYSIS CODING SCHEME}

1. Shows solidarity, raises other's status, gives help, reward. Examples: I think we did a great job!; I really like the example you used; thank you.

2. Shows tension release, jokes, laughs, shows satisfaction. Examples: Well, I guess I need to learn to spell!; lol; I'm sorry; apologies; explanation for not meeting an expectation.

3. Agrees, shows passive acceptance, understand, concurs, complies, confirms others opinions or analysis. Examples: I agree with what you have said, I know what you mean; mention of another person or the group in agreeing.

4. Gives suggestion or direction, implying autonomy for others; we use this category for procedural statements about the task or assignment at hand. Examples: Here is what we need to do next; I suggest we leave this question and come back; maybe we should do this in a chat room.

5. Gives opinion, evaluation, analysis, expresses feelings, wishes. These statements are about feelings or opinions on the task. Example: my analysis was; I think; personal examples.

6. Gives orientation, information, repeats, clarifies, confirms. These are facts or supported information from a source (observable) or the text about the content of the task. Example: the text said $X$; the definition of this was $X$; expressing agreement with the book; citing examples from the book.

7. Asks for orientation, information, repetition, confirmation. These questions are similar to those in Category 6 in that they are questions about facts and supported (observable) information. Examples: How does the book define $X$; didn't the text say this; the definition, the statement, or the question was $X$.

8. Asks for opinion, evaluation, analysis, expression of feeling. This category is similar to 5, except that it encompasses asking for these things rather than offering them. These questions may be directed to individuals or the group as a whole. Examples: what do you think about; how do you feel; what did you think when reading; what is your take on this, what do you think? 
9. Asks for suggestions, direction, possible ways of action. This category is similar to 4 in that we have determined these are questions about procedures, clarifying the task and asking how to accomplish the task. Examples: are we doing this right; should we do this in a chat room; when do we have to have this done?

10. Disagrees, shows passive rejection, formality, withholds help. These comments show negative social emotional reactions. Examples: I can't agree to that idea; I don't believe you are correct in that remark; I don't have anything to say about that.

11. Shows tension, asks for help, withdraws out of field. Examples: I don't think we can get this done in time; I don't know how we are supposed to do this without more help.

12. Shows antagonism, deflates another's status, defends or asserts self. Examples: Since I'm a mother and you are not, I think I would know; I can't believe anyone would make the comments you made.

Note: Categories 1 through 3 and 10 through 12 are about relational group work (helping to sustain or weaken relational ties within the group). Categories 4 and 9 are about procedures and how to go about completing the task. Categories 5 through 8 are content-related comments or questions. 